\title{
A evolução do mercado farmacêutico brasileiro no tratamento do glaucoma nos últimos 30 anos
}

\author{
Evolution of the Brazilian pharmaceutical market regardingglaucoma treatment \\ during the last 30 years
}

\author{
João Pessoa de Souza Filho ${ }^{1}$ \\ Ana Beatriz Toledo Dias ${ }^{2}$ \\ Acácio Alves de Souza Lima Filho ${ }^{3}$ \\ Marta Filippi Sartori ${ }^{4}$ \\ Maria Cristina Martins 5
}

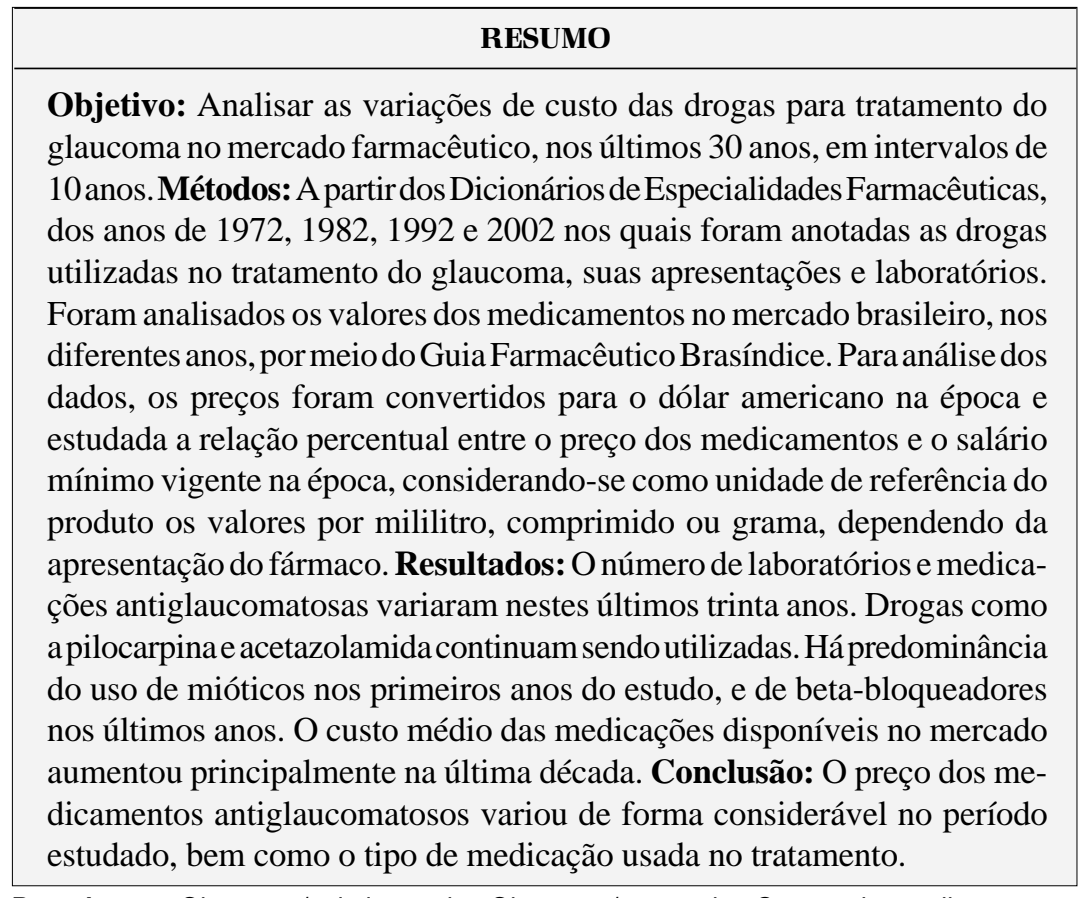

Descritores: Glaucoma/quimioterapia; Glaucoma/economia; Custos de medicamentos; Industria farmacêutica; Soluções oftálmicas/economia; Anti-hipertensivos/uso terapêutico

Trabalho realizado no Departamento de Oftalmologia da Universidade Federal de São Paulo - UNIFESP. ${ }^{1}$ Médico Oftalmologista, estagiário do setor de Catarata e Patologia Ocular do Departamento de Oftalmologia da Universidade Federal de São Paulo - UNIFESP.

${ }^{2}$ Tecnóloga em Oftalmologia, associada ao setor de Farmacologia e Patologia Ocular do Departamento de Oftalmologia da Universidade Federal de São Paulo - UNIFESP.

${ }^{3}$ Chefe do setor de Farmacologia Ocular do Departamento de Oftalmologia da Universidade Federal de São Paulo - UNIFESP.

${ }^{4}$ Professora Adjunta, Chefe da Disciplina de Oftalmologia da Faculdade de Medicina de Jundiaí.

Professora Adjunta da Disciplina de Oftalmologia da Faculdade de Medicina de Jundiaí e Chefe do Setor de Patologia Ocular do Departamento de Oftalmologia da Universidade Federal de São Paulo - UNIFESP.

Endereço para correspondência: Rua Manoel de Carvalho 287/602 - Recife (PE) CEP 52050-370

E-mail:jpessoa@oftalmo.epm.br

Recebido para análise em 26.09.2002

Versão revisada recebida em 14.01.2003

Aprovação em 07.04.2003

\section{INTRODUÇ̃̃̃O}

O glaucoma inclui um grupo de condições oculares caracterizado pela perda do campo visual e freqüentemente atribuído a um aumento da pressão intra-ocular ${ }^{(1)}$. O glaucoma é um problema de saúde pública e uma das mais importantes causas de cegueira no Brasil e no mundo. Nos Estados Unidos, 2,25 milhões de pessoas maiores de 45 anos têm glaucoma ${ }^{(2)}$. Segundo a OMS, a incidência de glaucoma no mundo é estimada em 2,4 milhões de casos por ano, e a prevalência de cegueira por glaucoma é de 5,2 milhões de pessoas, representando a terceira causa de cegueira no mundo ${ }^{(2-3)}$.

O mercado farmacêutico oftalmológico mundial atinge cerca de 4,6 bilhões de dólares, sendo que mais da metade deste valor está relacionado com terapias crônicas, principalmente com tratamento do glaucoma ${ }^{(4)}$.

O custo para o tratamento dessa doença é alto, somente os Estados Unidos gastam pelo menos US\$ 94 milhões por ano no tratamento do glaucoma $^{(3)}$.

Os agentes antiglaucomatosos são classificados em diversos grupos de acordo com sua estrutura química e seu mecanismo de ação ${ }^{(5-6)}$.
} 
Como toda doença crônica, o glaucoma apresenta problemas de aderência ao tratamento, que é influenciada por vários fatores, entre eles o custo dos medicamentos, principalmente em populações de baixa renda ${ }^{(3)}$.

Um estudo sobre o custo diário do tratamento do glaucoma demonstrou que existem inúmeras diferenças no custo dos colírios $^{(7)}$. E que diferenças no volume e número de gotas pode também levar a menor duração e maior custo para os usuários ${ }^{(8)}$.

Nos últimos anos, houve um aumento considerável do arsenal terapêutico graças à introdução de novas drogas hipotensoras ${ }^{(9)}$, dando ao médico uma variedade de escolhas terapêuticas que, se por um lado beneficia o paciente com um melhor controle da doença, por outro, tendem a elevar o custo do tratamento, dificultando a fidelidade ao tratamento ${ }^{(3)}$.

Alguns estudos analisam a influência do custo da medicação na eficácia do tratamento do glaucoma, porém, em nenhum estudo a evolução do custo do tratamento do glaucoma no Brasil nas últimas décadas foi analisada. Este estudo tem como objetivo analisar as variações de custo das drogas para tratamento do glaucoma, no mercado farmacêutico brasileiro, nos últimos 30 anos.

\section{MÉTODOS}

Foram selecionados os Dicionários de Especialidades Farmacêuticas (DEF), dos anos de $1972^{(10)}, 1982^{(11)}, 1992^{(12)}$ e $2002^{(13)}$ de onde foram anotadas as drogas utilizadas no tratamento do glaucoma, observando sua forma farmacêutica,

\begin{tabular}{|c|c|c|}
\hline & Salário mínimo - Brasil/US\$ dólar & Cotação - US\$ dólar \\
\hline 1972 & Cr\$ 268,80 / US\$ 45,10 & 1 US\$ - 5,96 Cr\$ \\
\hline 1982 & Cr\$ $16.608,00$ / US\$ 101,16 & 1 US\$ - 164,17 Cr\$ \\
\hline 1992 & $\mathrm{Cr} \$ 230.000,00 /$ US\$ 54,70 & 1 US\$ - 4204,60 Cr\$ \\
\hline 2002 & $\mathrm{R} \$ 200,00 /$ US\$ 58,47 & 1 US\$ - 3,42R\$ \\
\hline \multicolumn{3}{|c|}{ Fontes: Banco Central do Brasil, DIEESE, www.ai.com.br } \\
\hline
\end{tabular}

apresentação e concentrações, bem como os seus respectivos laboratórios.

Após a identificação das drogas existentes nos diferentes anos, foram consultados os valores dos medicamentos no mercado brasileiro, utilizando o Guia Farmacêutico Brasíndice ${ }^{(14)}$.

Para corrigir distorções com relação às diferentes moedas utilizadas em cada época, e assim realizar-se comparações, os preços foram convertidos para o dólar americano, cujo valor utilizado foi o câmbio médio oficial de venda divulgado pelo Banco Central do Brasil (BC) (Tabela 1). Também foi analisada a relação percentual entre o preço dos medicamentos e o salário mínimo vigente na época.

O preço de cada medicamento foi calculado por mililitros (preço/ml) se colírio, por grama (preço/g) se pomada e por comprimido ou drágea se as drogas eram de uso oral, para uniformizar os dados e facilitar a análise dos mesmos. Não foram incluídos neste trabalho medicamentos de uso parenteral.

\section{RESULTADOS}

Em 1972 foram encontrados 5 laboratórios produzindo 27 medicamentos utilizados no tratamento do glaucoma. Em 1982 eram 8 laboratórios e 34 medicamentos. Em 1992 existiam 4 laboratórios e 14 medicamentos, e em 2002, 9 laboratórios e 32 medicamentos (Tabela 2).

Dos 5 laboratórios presentes em 1972, 3 também estavam presentes no ano de 1982 (Alcon ${ }^{\circledR}$, Frumtost $^{\circledR}$ e Lederle $^{\circledR}$ ). O LOK $^{\circledR}$ estava associado com o Allergan ${ }^{\circledR}$, formando o AllerganLOK $^{\circledR}$. Neste ano encontramos 4 novos laboratórios produzindo medicamentos antiglaucomatosos: Merck Sharp Dohme ${ }^{\circledR}$ (MSD), Dover ${ }^{\circledR}$, Orbitron $^{\circledR}$ e Ayerst $^{\circledR}$ (Tabela 2).

Em 1992 encontramos os laboratórios Allergan-LOK ${ }^{\circledR}$, Alcon $^{\circledR}$, Frumtost ${ }^{\circledR}$ e MSD $^{\circledR}$.

Em 2002 os laboratórios em atividade são: Alcon $^{\circledR}, \mathrm{MSD}^{\circledR} \mathrm{e}$ Allergan $^{\circledR}$ que aparece associado ao Frumstost ${ }^{\circledR}$. Os novos laboratórios neste ano são: Pharmacia ${ }^{\circledR},{\mathrm{B} \& L^{\circledR}}^{\circledR}$, Wyeth ${ }^{\circledR}$, Novartis ${ }^{\circledR}$ e Ciba Vision ${ }^{\circledR}$ (Tabela 2).

\begin{tabular}{|c|c|c|c|c|c|c|c|}
\hline \multicolumn{2}{|c|}{1972} & \multicolumn{2}{|l|}{1982} & \multicolumn{2}{|l|}{1992} & \multicolumn{2}{|c|}{2002} \\
\hline Laboratórios & $\begin{array}{c}\mathrm{N}^{\circ} \\
\text { drogas }\end{array}$ & Laboratórios & $\begin{array}{c}\mathrm{N}^{\circ} \\
\text { drogas }\end{array}$ & Laboratórios & $\begin{array}{c}\mathrm{N}^{\circ} \\
\text { drogas }\end{array}$ & Laboratórios & $\begin{array}{c}\mathrm{N}^{\circ} \\
\text { drogas }\end{array}$ \\
\hline LOK & $9(33,3 \%)$ & Allergan-LOK & $10(29,5 \%)$ & Allergan-LOK & $5(35,8 \%)$ & Allergan & $10(31,3 \%)$ \\
\hline Alcon & $7(25,9 \%)$ & Alcon & $7(20,5 \%)$ & Alcon & $4(28,3 \%)$ & Alcon & $9(28,2 \%)$ \\
\hline Frumtost & $7(25,9 \%)$ & Frumtost & $7(20,5 \%)$ & Frumtost & $2(14,3 \%)$ & Pharmacia & $2(6,3 \%)$ \\
\hline Darrow & $3(11,2 \%)$ & MSD & $2(5,9 \%)$ & MSD/Chibret & $3(21,6 \%)$ & MSD & $6(18,7 \%)$ \\
\hline Lederle & $1(3,7 \%)$ & Dover & $3(8,9 \%)$ & - & - & $B \& L$ & $1(3,1 \%)$ \\
\hline- & - & Orbitron & $3(8,9 \%)$ & - & - & Wyeth & $1(3,1 \%)$ \\
\hline- & - & Lederle & $1(2,9 \%)$ & - & - & Novartis & $1(3,1 \%)$ \\
\hline- & - & Ayerst & $1(2,9 \%)$ & - & - & Cristália & $1(3,1 \%)$ \\
\hline- & - & - & - & - & - & Ciba Vision & $1(3,1 \%)$ \\
\hline Total 5 & 27 (100\%) & Total 8 & 34 (100\%) & Total 4 & $14(100 \%)$ & Total 9 & $32(100 \%)$ \\
\hline Fonte: $\mathrm{DEF}^{(10-13)}$ & & & & & & & \\
\hline
\end{tabular}


Classificando-se o número de medicamentos por grupo farmacológico, encontrou-se no ano de 1972 uma predominância de mióticos $(77,6 \%)$, seguido dos inibidores da anidrase carbônica e associações de drogas (Epilo ${ }^{\circledR}$ 2\% e 4\% e Glauco$\sin ^{\circledR}$ ) com $11,2 \%$ cada.

Em 1982 ainda predominavam os mióticos (52,9\%), representados basicamente pela pilocarpina. Os agonistas adrenérgicos representavam 17,6\% e os beta-bloqueadores e os inibidores da anidrase carbônica correspondiam, cada um, a 11,8\% das drogas antiglaucomatosas produzidas. As associações de drogas representavam 5,9\% dos medicamentos deste ano.

Em 1992, os beta-bloqueadores predominavam, representando $50 \%$ das drogas disponíveis no mercado, enquanto os mióticos passaram a representar 35,8\% das drogas hipotensoras do mercado (Tabela 3).

$\mathrm{Na}$ atualidade observa-se preponderância dos beta-bloqueadores $(46,8 \%)$ entre os medicamentos. Os mióticos representam $15,6 \%$ das drogas utilizadas. Observa-se ainda o aparecimento de novas drogas para o tratamento do glaucoma (Tabela 3).

Quando se observa o preço dos medicamentos comerciais em moeda brasileira nos diferentes anos e utiliza-se a conversão para o dólar americano por unidade do produto (P US\$), observou-se uma variação no preço dos medicamentos e da relação com o salário mínimo vigente nos diferentes anos. (Tabelas 4, 5, 6, 7)

\section{DISCUSSÃO}

A terapêutica empregada no glaucoma envolve um número limitado de drogas. Entretanto, existe atualmente, um maior arsenal terapêutico com um maior número de laboratórios. Em 1972 existiam apenas 5 laboratórios que produziam 27 diferentes medicamentos antiglaucomatosos, sendo que nesta época o laboratório LOK ${ }^{\circledR}$ predominava produzindo 33,3\% dos produtos. Observou-se ainda apenas 2 tipos de grupos farmacológicos principais: os mióticos e os inibidores da anidrase carbônica de uso oral.

Em 1982 o número de laboratórios que produziam medicamentos hipotensores oculares aumentou para 8, os quais pro- duziam 34 medicamentos. Somente o laboratório AllerganLOK $^{\circledR}$ foi responsável pela produção de 29,5\% das drogas deste ano. Observou-se também em 1982 um maior número de drogas existentes no mercado quando comparado com as existentes em 1972. Sendo que os mióticos eram responsáveis por 52,9\% dos medicamentos disponíveis no mercado.

Em 1992 observou-se uma redução no número de laboratórios: apenas 4 produziam 14 medicamentos. Mesmo assim o Laboratório Allergan-LOK ${ }^{\circledR}$ continuava a ter o maior número de produtos disponíveis no mercado farmacêutico (35,8\%). Neste ano já se observou uma predominância dos beta-bloqueadores, representando 50,0\% dos medicamentos produzidos e os mióticos representavam apenas 35,8\% das drogas.

Em 2002 tanto o número de laboratórios, quanto de drogas antiglaucomatosas voltou a aumentar, foram observados 9 laboratórios farmacêuticos e 32 medicamentos, com predominância do Laboratório Allergan-Frumtost ${ }^{\circledR}$ (31,3\%). Neste ano tem-se uma predominância dos beta-bloqueadores (46,8\%). Novas drogas surgiram, como os inibidores da anidrase carbônica de uso tópico, agentes agonistas adrenérgicos seletivos e os análogos de prostaglandinas.

Observou-se que algumas drogas tais como: dimetilbrometo de decametileno, carbaminoilcolina, iodedo de fosfolina, epinefrina, carbacol e a diclorfenamida, além das associações de adrenalina e pilocarpina e acetazolamida com meprobamato não estão mais disponíveis no mercado oftalmológico atual. Outras drogas como pilocarpina e acetazolamida continuam atuais, fazendo parte do arsenal terapêutico do glaucoma ainda nos dias de hoje.

Notou-se que as grandes companhias multinacionais como a Alcon ${ }^{\circledR}$, a Allergan ${ }^{\circledR}$ (LOK e Frumtost) e MSD ${ }^{\circledR}$, praticamente dominaram o mercado das drogas antiglaucomatosas nos últimos 30 anos, e que alguns laboratórios, tais como a Darrow $^{\circledR}$, Dover ${ }^{\circledR}$, Ayerst ${ }^{\circledR}$, Orbitron ${ }^{\circledR}$ e Lederle ${ }^{\circledR}$, não mais produzem medicamentos para o tratamento do glaucoma.

Silva et $\mathrm{al}^{(15)}$, em estudo atual, observaram que os medicamentos mais utilizados no tratamento clínico do glaucoma foram os beta-bloqueadores $(97,3 \%)$, resultado também observado por Amaral Filho et $\mathrm{al}^{(3)}$ em estudo sobre custo mensal de medicações antiglaucomatosas no Brasil. No presente estudo

\begin{tabular}{|c|c|c|c|c|}
\hline Grupo farmacológico & $\begin{array}{c}1972 \\
\mathrm{~N}^{\circ} \text { drogas }\end{array}$ & $\begin{array}{c}1982 \\
\mathrm{~N}^{\circ} \text { drogas }\end{array}$ & $\begin{array}{c}1992 \\
\mathrm{~N}^{\circ} \text { drogas }\end{array}$ & $\begin{array}{c}2002 \\
\mathrm{~N}^{\circ} \text { drogas }\end{array}$ \\
\hline Beta-bloqueadores & - & $4(11,8 \%)$ & $7(50,0 \%)$ & $15(46,8 \%)$ \\
\hline Mióticos & $21 \quad(77,6 \%)$ & $18(52,9 \%)$ & $5(35,8 \%)$ & $5(15,6 \%)$ \\
\hline Agonista adrenérgicos & - & $6(17,6 \%)$ & $1(7,1 \%)$ & $3(9,4 \%)$ \\
\hline Inibidores da anidrase carbônica & $3(11,2 \%)$ & $4(11,8 \%)$ & $1(7,1 \%)$ & $3(9,4 \%)$ \\
\hline Prostaglandinas e Prostamidas & - & - & - & $4(12,5 \%)$ \\
\hline Associações & $3(11,2 \%)^{* 1}$ & $2(5,9 \%)^{* 2}$ & - & $2(6,3 \%)^{\star 3}$ \\
\hline Total & $27(100 \%)$ & $34(100 \%)$ & $14(100 \%)$ & $32(100 \%)$ \\
\hline \multicolumn{5}{|c|}{$\begin{array}{l}{ }^{*}{ }_{1} \text { Epilo }{ }^{\oplus} \text { (Pilocarpina e Epinefrina) } 2 \% \text { e } 4 \% \text { e Glaucosin }{ }^{\oplus} \text { (Acetazolamida e Mepobramato); }{ }^{* 2} \text { Epilo }^{\oplus} 2 \% \text { e } 4 \%{ }^{*}{ }^{* 3} \text { Cosopt }^{\oplus} \text { (Timolol e Dorzolamida) e Xalacon }{ }^{\oplus} \text { (Timolo } \\
\text { Fonte: DEF }{ }^{(10-13)}\end{array}$} \\
\hline
\end{tabular}




\begin{tabular}{|c|c|c|c|c|c|c|c|}
\hline Droga & Laboratório & C & $\mathbf{Q}$ & $\mathbf{P}$ & PT US\$ & P US\$ & $\%$ SM \\
\hline \multirow[t]{18}{*}{ Pilocarpina } & \multirow[t]{6}{*}{ Alcon $^{\circledR}$} & $1 \%$ & $15 \mathrm{ml}$ & 9,98 & 1,67 & 0,11 & $0,24 \%$ \\
\hline & & $2 \%$ & $15 \mathrm{ml}$ & 11,79 & 1,97 & 0,13 & $0,29 \%$ \\
\hline & & $3 \%$ & $15 \mathrm{ml}$ & 12,99 & 2,17 & 0,14 & $0,31 \%$ \\
\hline & & $4 \%$ & $15 \mathrm{ml}$ & 13,38 & 2,24 & 0,15 & $0,33 \%$ \\
\hline & & $2 \%$ & $10 \mathrm{ml}$ & 8,40 & 1,40 & 0,14 & $0,31 \%$ \\
\hline & & $4 \%$ & $10 \mathrm{ml}$ & 9,68 & 1,62 & 0,16 & $0,35 \%$ \\
\hline & \multirow[t]{6}{*}{ LOK $^{\circledR}$} & $2 \%$ & $5 \mathrm{ml}$ & 7,32 & 1,22 & 0,24 & $0,53 \%$ \\
\hline & & $4 \%$ & $5 \mathrm{ml}$ & 7,96 & 1,33 & 0,26 & $0,58 \%$ \\
\hline & & $2 \%$ & $5 \mathrm{ml}$ & 6,25 & 1,04 & 0,20 & $0,44 \%$ \\
\hline & & $4 \%$ & $5 \mathrm{ml}$ & 7,18 & 1,20 & 0,24 & $0,53 \%$ \\
\hline & & $2 \%$ & $3,5 \mathrm{~g}$ & 5,78 & 0,96 & 0,27 & $0,60 \%$ \\
\hline & & $4 \%$ & $3,5 \mathrm{~g}$ & 6,78 & 1,13 & 0,32 & $0,71 \%$ \\
\hline & \multirow[t]{3}{*}{ Frumtost $^{\circledR}$} & $2 \%$ & $5 \mathrm{ml}$ & 3,51 & 0,58 & 0,11 & $0,24 \%$ \\
\hline & & $4 \%$ & $5 \mathrm{ml}$ & 5,57 & 0,93 & 0,18 & $0,40 \%$ \\
\hline & & $6 \%$ & $5 \mathrm{ml}$ & 5,90 & 0,98 & 0,19 & $0,42 \%$ \\
\hline & \multirow[t]{3}{*}{ Darrow $^{\circledR}$} & $1 \%$ & $15 \mathrm{ml}$ & 8,53 & 1,43 & 0,09 & $0,20 \%$ \\
\hline & & $2 \%$ & $15 \mathrm{ml}$ & 10,24 & 1,71 & 0,11 & $0,24 \%$ \\
\hline & & $4 \%$ & $15 \mathrm{ml}$ & 11,94 & 2,00 & 0,13 & $0,29 \%$ \\
\hline \multirow[t]{2}{*}{ Decametileno*1 } & \multirow[t]{2}{*}{ Frumtost $^{\circledast}$} & $0,125 \%$ & $3 \mathrm{ml}$ & 2,02 & 0,33 & 0,11 & $0,24 \%$ \\
\hline & & $0,25 \%$ & $3 \mathrm{ml}$ & 2,50 & 0,41 & 0,13 & $0,29 \%$ \\
\hline Carbaminoilcolina & LOK$^{\circledR}$ & - & $5 \mathrm{ml}$ & 4,05 & 0,67 & 0,13 & $0,29 \%$ \\
\hline \multirow[t]{2}{*}{ Acetazolamida } & Lederle $^{\circledast}$ & $250 \mathrm{mg}$ & $25 \mathrm{cp}$ & 6,24 & 1,04 & 0,04 & $0,08 \%$ \\
\hline & LOK $^{\circledast}$ & $250 \mathrm{mg}$ & $20 \mathrm{cp}$ & 10,99 & 1,84 & 0,09 & $0,20 \%$ \\
\hline Diclorfenamida & $\mathrm{LOK}^{\circledR}$ & $50 \mathrm{mg}$ & $20 \mathrm{cp}$ & 16,52 & 2,77 & 0,13 & $0,29 \%$ \\
\hline \multirow[t]{3}{*}{ Associação } & Frumtost ${ }^{\circledR}$ & $2 \% \star 2$ & $5 \mathrm{ml}$ & 4,86 & 0,81 & 0,16 & $0,35 \%$ \\
\hline & & $4 \% * 2$ & $5 \mathrm{ml}$ & 5,67 & 0,95 & 0,19 & $0,42 \%$ \\
\hline & & $\star 3$ & $20 \mathrm{cp}$ & 11,04 & 1,85 & 0,09 & $0,20 \%$ \\
\hline
\end{tabular}

os beta-bloqueadores apresentavam um custo médio de: US\$1,83/ml para o ano de 1982; US\$ 0,43/ml em 1992 e US\$ 0,66/ $\mathrm{ml}$, no ano de 2002, demonstrando um decréscimo no custo do tratamento com este tipo de medicamento. O que pode justificar o aumento do preço no ano de 2002, é o aparecimento de novas apresentações da droga como o timolol $\mathrm{XE}^{\circledR}$, betaxolol $\mathrm{S}^{\circledR}$ e o metipranolol.

Observou-se ainda neste estudo, que drogas com mesma concentração, porém de diferentes laboratórios, apresentavam variação de preços, como é o caso da pilocarpina a $2 \%$, que em 1972, fabricada pela Darrow ${ }^{\circledR}$ custava US\$ 0,11/ml, e a produzida pelo $\mathrm{LOK}^{\circledR}$ era vendida a US\$ $0,24 / \mathrm{ml}$, uma diferença de preço de US\$ 0,13 por ml (118\%), já a essa mesma droga em 2002 custava de US\$ 0,24/ml (Alcon ${ }^{\circledR}$ ) a US\$ 0,34/ml (Aller$\left.\operatorname{gan}^{\circledR}\right)$, uma diferença de US\$ 0,10 por $\mathrm{ml}(41,7 \%)$, com isso nota-se que essa droga, atualmente, mantém diferentes preços dependendo do laboratório, porém, está mais cara do que há 30 anos.

Algumas drogas como a acetazolamida tiveram nos últimos trinta anos um aumento de preço tanto se utilizando o dólar americano (US\$) por comprimido, como se utilizando o salário mínimo como referência. Na comparação com o dólar americano o aumento de custo foi de $125 \%$ (US\$ 0,04 a 0,09), e na comparação com o salário mínimo de $87 \%$ (0,08\% a 0,15\%) no período analisado (1972 - 2002).

Para exemplificar a evolução do custo dos medicamentos antiglaucomatosos, de uma maneira geral, tomamos a liberdade de calcular o custo médio dos colírios (que são a grande maioria das drogas disponíveis no mercado) que passamos a descrever: a)1972: US\$ 0,15/ml, b)1982: US \$ 0,43/ml, c) 1992: US\$ 0,39/ml e d) 2002: US\$1,76/ml. Esses valores ficam fáceis de serem analisados quando se observa o Gráfico 1.

Atualmente é possível encontrar drogas que custam por mililitro até $16 \%$ do salário mínimo (US\$ 9,36/ml). Talvez por isso, medicamentos com preço mais baixo, como maleato de timolol, sejam ainda, no Brasil, droga de primeira escolha para o tratamento do glaucoma por apresentar a melhor relação custo/benefício.

É importante salientar que não computamos nos nossos cálculos as taxas inflacionárias sobre o dólar americano ${ }^{(16)}$.

Os preços dos fármacos lançados nos últimos anos estão muito acima da capacidade de compra do cidadão que ganha atualmente um salário mínimo, dificultando o acesso dos mesmos aos avanços terapêuticos. Isto é particularmente verdade 


\begin{tabular}{|c|c|c|c|c|c|c|c|}
\hline Droga & Laboratório & C & $\mathbf{Q}$ & $\mathbf{P}$ & PT US\$ & P US\$ & $\%$ SM \\
\hline \multirow[t]{12}{*}{ Timolol } & $\mathrm{MSD}^{\circledR}$ & $0,25 \%$ & $5 \mathrm{ml}$ & $1.385,00$ & 8,43 & 1,68 & $1,67 \%$ \\
\hline & & $0,50 \%$ & $5 \mathrm{ml}$ & $1.629,00$ & 9,92 & 1,98 & $1,96 \%$ \\
\hline & Frumtost ${ }^{\circledR}$ & $0,25 \%$ & $5 \mathrm{ml}$ & $1.385,00$ & 8,43 & 1,68 & $1,67 \%$ \\
\hline & & $0,50 \%$ & $5 \mathrm{ml}$ & $1.629,00$ & 9,92 & 1,98 & $1,96 \%$ \\
\hline & Alcon $^{\circledast}$ & $2 \%$ & $10 \mathrm{ml}$ & 290,00 & 1,76 & 0,17 & $0,17 \%$ \\
\hline & & $2 \%$ & $15 \mathrm{ml}$ & 366,00 & 2,22 & 0,14 & $0,14 \%$ \\
\hline & Allergan LOK ${ }^{\circledR}$ & $3 \%$ & $15 \mathrm{ml}$ & 402,00 & 2,44 & 0,16 & $0,16 \%$ \\
\hline & & $4 \%$ & $15 \mathrm{ml}$ & 414,00 & 2,52 & 0,17 & $0,17 \%$ \\
\hline & & $1 \%$ & $15 \mathrm{ml}$ & 290,00 & 1,76 & 0,11 & $0,11 \%$ \\
\hline & & $2 \%$ & $5 \mathrm{ml}$ & 212,00 & 1,29 & 0,25 & $0,25 \%$ \\
\hline & & $4 \%$ & $5 \mathrm{ml}$ & 232,00 & 1,41 & 0,28 & $0,28 \%$ \\
\hline & & $2 \%$ & $5 \mathrm{ml}$ & 196,00 & 1,19 & 0,23 & $0,23 \%$ \\
\hline \multirow[t]{8}{*}{ Pilocarpina } & Frumtost ${ }^{\circledR}$ & $4 \%$ & $5 \mathrm{ml}$ & 223,00 & 1,35 & 0,27 & $0,27 \%$ \\
\hline & $* 1$ & $4 \%$ & $3,5 \mathrm{~g}$ & - & - & - & - \\
\hline & & $2 \%$ & $5 \mathrm{ml}$ & 101,00 & 0,61 & 0,12 & $0,12 \%$ \\
\hline & Dover $^{\circledR}$ & $4 \%$ & $5 \mathrm{ml}$ & 169,00 & 1,02 & 0,20 & $0,20 \%$ \\
\hline & & $6 \%$ & $5 \mathrm{ml}$ & 162,00 & 0,98 & 0,19 & $0,19 \%$ \\
\hline & & $1 \%$ & $15 \mathrm{ml}$ & 294,00 & 1,79 & 0,11 & $0,11 \%$ \\
\hline & & $2 \%$ & $15 \mathrm{ml}$ & 355,00 & 2,16 & 0,14 & $0,14 \%$ \\
\hline & & $4 \%$ & $15 \mathrm{ml}$ & 414,00 & 2,52 & 0,16 & $0,16 \%$ \\
\hline Carbacol & Alcon $^{\circledast}$ & $3 \%$ & $15 \mathrm{ml}$ & 490,00 & 2,98 & 0,19 & $0,19 \%$ \\
\hline Fosfolina & Ayerst $^{\circledR * 2}$ & $3 \mathrm{mg}$ & - & 922,00 & 5,61 & - & - \\
\hline \multirow[t]{2}{*}{ Acetazolamida } & Lederle $^{\circledR}$ & $250 \mathrm{mg}$ & $25 \mathrm{cp}$ & 490,00 & 2,98 & 0,11 & $0,11 \%$ \\
\hline & Allergan-LOK & $250 \mathrm{mg}$ & $20 \mathrm{cp}$ & 247,00 & 1,50 & 0,07 & $0,07 \%$ \\
\hline \multirow[t]{2}{*}{ Diclorfenamida } & Alcon ${ }^{\circledast}$ & $50 \mathrm{mg}$ & $25 \mathrm{cp}$ & 564,00 & 3,43 & 0,13 & $0,13 \%$ \\
\hline & Allergan-LOK & $50 \mathrm{mg}$ & $20 \mathrm{cp}$ & 312,00 & 1,90 & 0,09 & $0,09 \%$ \\
\hline \multirow[t]{6}{*}{ L Epinefrina } & Allergan-LOK & $0,50 \%$ & $5 \mathrm{ml}$ & 227,00 & 1,38 & 0,27 & $0,27 \%$ \\
\hline & & $1 \%$ & $5 \mathrm{ml}$ & 242,00 & 1,47 & 0,29 & $0,29 \%$ \\
\hline & & $2 \%$ & $5 \mathrm{ml}$ & 270,00 & 1,64 & 0,32 & $0,32 \%$ \\
\hline & Orbitron $^{\circledR}$ & $0,50 \%$ & $5 \mathrm{ml}$ & 189,00 & 1,15 & 0,23 & $0,32 \%$ \\
\hline & & $1 \%$ & $5 \mathrm{ml}$ & 236,00 & 1,43 & 0,28 & $0,28 \%$ \\
\hline & & $2 \%$ & $5 \mathrm{ml}$ & 254,00 & 1,54 & 0,30 & $0,30 \%$ \\
\hline \multirow[t]{2}{*}{ Associação } & Frumtost ${ }^{\circledR \star 3}$ & $2 \%$ & $5 \mathrm{ml}$ & 122,00 & 0,74 & 0,14 & $0,14 \%$ \\
\hline & & $4 \%$ & $5 \mathrm{ml}$ & 139,00 & 0,84 & 0,16 & $0,16 \%$ \\
\hline
\end{tabular}

\begin{tabular}{|c|c|c|c|c|c|c|c|}
\hline \multicolumn{8}{|c|}{$\begin{array}{l}\text { Tabela 6. Apresentação das drogas disponíveis no mercado farmacêutico relacionando: (C) concentração, (Q) quantidade do produto na } \\
\text { apresentação comercial, (P) preço da apresentação comercial em Cruzeiros, (PT US\$) preço da apresentaça comercial em dólar americano, } \\
\text { (P US\$) preço em dólar americano por ml/cp/g e (\% SM) relação percentual entre o preço em dólar americano por ml/cp/g com o salário mínimo. } \\
\text { Ano } 1992\end{array}$} \\
\hline Droga & Laboratório & C & $\mathbf{Q}$ & $\mathbf{P}$ & PT US\$ & P US\$ & $\%$ SM \\
\hline \multirow[t]{5}{*}{ Timolol } & Alcon $^{\circledast}$ & $0,50 \%$ & $5 \mathrm{ml}$ & $7.071,00$ & 1,68 & 0,33 & $0,60 \%$ \\
\hline & $\mathrm{MSD}^{\circledR}$ & $0,25 \%$ & $5 \mathrm{ml}$ & $7.895,00$ & 1,87 & 0,37 & $0,68 \%$ \\
\hline & Frumtost $^{\circledR}$ & $0,50 \%$ & $5 \mathrm{ml}$ & $9.089,00$ & 2,16 & 0,43 & $0,79 \%$ \\
\hline & & $0,25 \%$ & $5 \mathrm{ml}$ & $6.550,00$ & 1,55 & 0,31 & $0,57 \%$ \\
\hline & & $0,50 \%$ & $5 \mathrm{ml}$ & $7.680,00$ & 1,82 & 0,36 & $0,66 \%$ \\
\hline Levobunolol & Allergan $^{\circledR}$ & $0,50 \%$ & $5 \mathrm{ml}$ & $11.269,00$ & 2,68 & 0,53 & $0,97 \%$ \\
\hline Betaxolol & Alcon $^{\circledast}$ & $0,50 \%$ & $5 \mathrm{ml}$ & $14.479,00$ & 3,44 & 0,68 & $1,24 \%$ \\
\hline Acetazolamida & $\mathrm{MSD}^{\circledR}$ & $250 \mathrm{mg}$ & $25 \mathrm{cp}$ & $11.835,00$ & 2,81 & 0,11 & $0,20 \%$ \\
\hline \multirow[t]{5}{*}{ Pilocarpina } & Alcon $^{\circledast}$ & $2 \%$ & $15 \mathrm{ml}$ & $15.490,00$ & 3,68 & 0,24 & $0,44 \%$ \\
\hline & & $4 \%$ & $15 \mathrm{ml}$ & $24.370,00$ & 5,79 & 0,38 & $0,70 \%$ \\
\hline & Allergan $^{\circledR}$ & $1 \%$ & $10 \mathrm{ml}$ & $10.210,00$ & 2,42 & 0,24 & $0,44 \%$ \\
\hline & & $2 \%$ & $10 \mathrm{ml}$ & $14.330,00$ & 3,40 & 0,34 & $0,62 \%$ \\
\hline & & $4 \%$ & $10 \mathrm{ml}$ & $22.330,00$ & 5,31 & 0,53 & $0,97 \%$ \\
\hline Dipivefrina & Allergan $^{\circledast}$ & & $10 \mathrm{ml}$ & $17.050,00$ & 4,05 & 0,40 & $0,73 \%$ \\
\hline
\end{tabular}




\begin{tabular}{|c|c|c|c|c|c|c|c|}
\hline Droga & Laboratório & C & $\mathbf{Q}$ & $\mathbf{P}$ & PT US\$ & P US\$ & $\%$ SM \\
\hline \multirow{12}{*}{ Timolol } & \multirow{2}{*}{ Alcon $^{\circledR}$} & $0,5 \%$ & $5 \mathrm{ml}$ & 4,82 & 1,40 & 0,28 & $0,48 \%$ \\
\hline & & $0,5 \%$ & $5 \mathrm{ml}$ & 4,49 & 1,31 & 0,26 & $0,44 \%$ \\
\hline & \multirow[t]{3}{*}{ Allergan ${ }^{\circledR}$} & $0,25 \%$ & $5 \mathrm{ml}$ & 4,97 & 1,45 & 0,29 & $0,49 \%$ \\
\hline & & $0,5 \%$ & $5 \mathrm{ml}$ & 5,88 & 1,75 & 0,34 & $0,58 \%$ \\
\hline & & $0,5 \%$ & $10 \mathrm{ml}$ & 11,80 & 3,45 & 0,34 & $0,58 \%$ \\
\hline & $* 1$ & $0,5 \%$ & $5 \mathrm{ml}$ & - & - & - & - \\
\hline & \multirow[t]{4}{*}{$\mathrm{MSD}^{\circledR}$} & $0,25 \%$ & $5 \mathrm{ml}$ & 6,62 & 1,93 & 0,38 & $0,65 \%$ \\
\hline & & $0,5 \%$ & $5 \mathrm{ml}$ & 7,86 & 2,29 & 0,45 & $0,77 \%$ \\
\hline & & $0,25 \%$ XE & $5 \mathrm{ml}$ & 22,80 & 6,66 & 1,33 & $2,27 \%$ \\
\hline & & $0,5 \% \times E$ & $5 \mathrm{ml}$ & 27,42 & 8,01 & 1,60 & $2,74 \%$ \\
\hline & Ciba Vision ${ }^{\circledR}$ & $0,1 \%$ & $5 \mathrm{ml}$ & 18,60 & 5,43 & 1,08 & $1,85 \%$ \\
\hline & Cristália $^{\circledR}$ & $0,5 \%$ & $5 \mathrm{ml}$ & 4,70 & 1,37 & 0,27 & $0,46 \%$ \\
\hline \multirow[t]{2}{*}{ Levobunolol } & \multirow[t]{2}{*}{ Allergan } & $0,5 \%$ & $5 \mathrm{ml}$ & 12,89 & 3,76 & 0,75 & $1,29 \%$ \\
\hline & & $0,5 \%$ & $10 \mathrm{ml}$ & 22,38 & 6,54 & 0,65 & $1,12 \%$ \\
\hline \multirow[t]{2}{*}{ Betaxolol } & \multirow[t]{2}{*}{ Alcon $^{\circledast}$} & $0,5 \%$ & $5 \mathrm{ml}$ & 12,19 & 3,56 & 0,71 & $1,21 \%$ \\
\hline & & $0,25 \% \mathrm{~S}$ & $5 \mathrm{ml}$ & 16,27 & 4,75 & 0,95 & $1,62 \%$ \\
\hline Metipranolol & $B \& L^{\circledR}$ & $0,3 \%$ & $5 \mathrm{ml}$ & 15,39 & 4,50 & 0,90 & $1,54 \%$ \\
\hline \multirow[t]{5}{*}{ Pilocarpina } & \multirow[t]{2}{*}{ Alcon $^{\circledast}$} & $2 \%$ & $15 \mathrm{ml}$ & 9,42 & 2,75 & 0,18 & $0,31 \%$ \\
\hline & & $4 \%$ & $15 \mathrm{ml}$ & 16,32 & 4,77 & 0,31 & $0,53 \%$ \\
\hline & \multirow[t]{3}{*}{ Allergan } & $1 \%$ & $10 \mathrm{ml}$ & 9,35 & 2,73 & 0,27 & $0,46 \%$ \\
\hline & & $2 \%$ & $10 \mathrm{ml}$ & 13,14 & 3,84 & 0,38 & $0,65 \%$ \\
\hline & & $4 \%$ & $10 \mathrm{ml}$ & 19,21 & 5,61 & 0,56 & $0,96 \%$ \\
\hline Acetazolamida & Wyeth ${ }^{\circledR}$ & $250 \mathrm{mg}$ & $25 \mathrm{cp}$ & 8,54 & 2,49 & 0,09 & $0,15 \%$ \\
\hline Dorzolamida & $\mathrm{MSD}^{\circledR}$ & $2 \%$ & $5 \mathrm{ml}$ & 36,61 & 10,70 & 2,14 & $3,66 \%$ \\
\hline Brinzolamida & Alcon $^{\circledR}$ & $1 \%$ & $5 \mathrm{ml}$ & 31,33 & 9,16 & 1,83 & $3,13 \%$ \\
\hline Dipivefrina & Allergan $^{\circledR}$ & & $10 \mathrm{ml}$ & 14,33 & 4,19 & 0,41 & $0,70 \%$ \\
\hline \multirow[t]{2}{*}{ Brimonidina } & \multirow[t]{2}{*}{ Allergan $^{\circledR}$} & $0,2 \%$ & $5 \mathrm{ml}$ & 38,80 & 11,34 & 2,26 & $3,86 \%$ \\
\hline & & $0,2 \%$ & $10 \mathrm{ml}$ & 62,69 & 18,33 & 1,83 & $3,13 \%$ \\
\hline Apraclonidina & Alcon $^{\circledR}$ & $0,5 \%$ & $5 \mathrm{ml}$ & 33,17 & 9,69 & 1,93 & $3,30 \%$ \\
\hline Travoprost & Alcon $^{\circledast}$ & $0,004 \%$ & $2,5 \mathrm{ml}$ & 60,93 & 17,81 & 7,12 & $12,18 \%$ \\
\hline Bimatoprost & Allergan & $0,03 \%$ & $3 \mathrm{ml}$ & 67,69 & 19,79 & 6,59 & $11,27 \%$ \\
\hline \multicolumn{8}{|l|}{ Unoprostona } \\
\hline Latanoprost & Pharmacia $^{\circledR}$ & $0,005 \%$ & $2,5 \mathrm{ml}$ & 71,28 & 20,84 & 8,33 & $14,25 \%$ \\
\hline \multirow[t]{2}{*}{ Associação } & $\mathrm{MSD}^{\circledR \star 2}$ & - & $5 \mathrm{ml}$ & 54,10 & 15,81 & 3,16 & $5,40 \%$ \\
\hline & Pharmacia $^{\circledR * 3}$ & - & $2,5 \mathrm{ml}$ & 80,06 & 23,40 & 9,36 & $16,00 \%$ \\
\hline
\end{tabular}

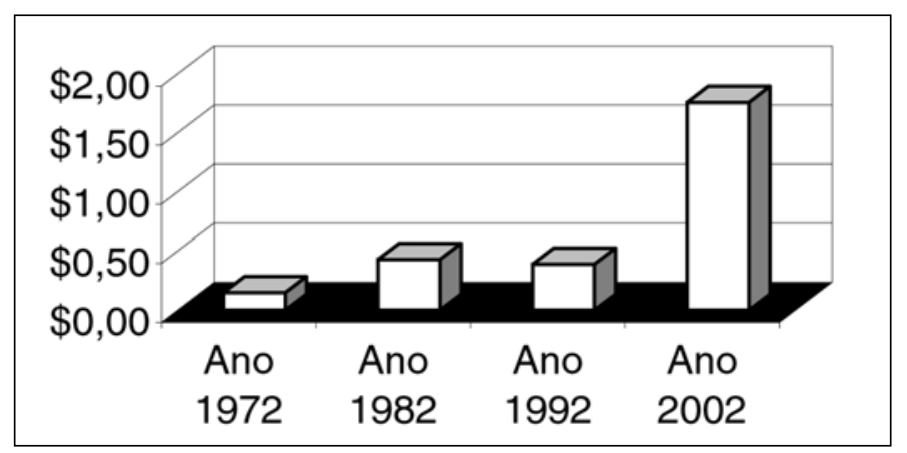

Gráfico 1. Custo médio dos colírios antiglaucomatosos (US $\$ / \mathrm{ml})$

em doenças crônicas como o glaucoma, na qual nem sempre se obtém um controle da doença com uma única droga.

O presente estudo nos permitiu observar a evolução da indústria farmacêutica no que diz respeito à diversidade de agentes farmacológicos e variação dos seus preços. Não se pode negar que o fator custo está diretamente relacionado a essa evolução. Esperamos que o Brasil consiga, pelo menos em médio prazo, disponibilizar para todos os novos recursos terapêuticos, cumprindo assim o que determina a Constituição Federal Brasileira, ou seja, que a saúde é um direito de todos e um dever do Estado (artigo 196).

\section{CONCLUSÕES}

Nos últimos trinta anos:

1- O número de laboratórios e medicamentos antiglaucomatosos variou, de 4 laboratórios e 14 drogas para 9 laboratórios e 32 medicamentos. 
2 - O Laboratório Alcon ${ }^{\circledR}$ foi o único a manter a produção de drogas antiglaucomatosas.

3 - Os grupos farmacológicos passaram de dois para cinco.

4 - Drogas como a pilocarpina e acetazolamida, utilizadas desde 1972, continuam atuais.

5 - A utilização dos mióticos predominou na primeira metade do estudo, decaindo consideravelmente de 77,6\% em 1972 para $15,6 \%$ na avaliação de 2002 .

6 - O uso dos beta-bloqueadores predominou na segunda metade do estudo.

7 - O custo médio da medicação hipotensora ocular variou ao longo destes anos estando consideravelmente maior nos últimos 10 anos.

8 - Não existem atualmente as pomadas oftálmicas antiglaucomatosas no mercado brasileiro e os mióticos limitados apenas a pilocarpina.

\section{ABSTRACT}

Purpose: To analyze price variations of drugs used to control glaucoma, during the last 30 years, at 10-year intervals. Methods: Drugs to treat glaucoma, as well as their presentations and laboratories were selected from the 1972, 1982, 1992 and 2002 editions of the Dictionary of Pharmaceutical Specialties. The price of medicines in the Brazilian market were analyzed, in the different decades, using the pharmaceutical guide "Brasíndice". For analysis of the data, the prices were converted to American dollars and the relationship between the medicine and the minimum wage was analyzed. As unit of the product, the values per milliliter, oral tablet or gram, depending on the presentation, were considered. Results: The number of laboratories and antiglaucoma medications varied in the last thirty years. Drugs such as acetazolamide and pilocarpine still continue to be used. Parasympathomimetic drugs have predominantly been used in the first years of the study, and the beta-blockers in the last years. The average cost of available medications in the market has increased in the last decade. Conclusion: The price of antiglaucoma drugs varied considerably in the studied period as well as the type of drug used in the treatment.
Keywords: Glaucoma/drug therapy; Glaucoma/economics; Drug costs; Drug industry; Ophthalmic solutions/economics; Antihypertensive agents/therapeutic use

\section{REFERÊNCIAS}

1. Walckiers D, Sartor F. Results of an epidemiological study on drug-treated intraocular hypertension in Belgium. J Clin Epidemiol 1996;49:489-93.

2. Wilson MR, Martone JF. Epidemilogy of chronic open angle glaucoma. In: Ritch R, Shields MB, Krupin T. The glaucomas. 3rd ed. St Louis: Mosby; 1996. p.407-45.

3. Amaral Filho JM, Moreira RAR, Silva LMS, Vasconcelos JP, Rocha EM, Costa VP, et al. Custo mensal de medicações antiglaucomatosas no Brasil. Arq Bras Oftalmol 1999;62:123-6.

4. Novack GD. Finacing New Drug Development in Ophthalmology. J Glaucoma 2000;9:195-9.

5. Arruda Mello PA, Moura CR. Tratamento clínico do glaucoma. In: Susanna Junior R. Glaucoma, manual básico. São Paulo: Cultura médica;1999. p.181-214.

6. Ventura MP, Dias JFP, Albuquerque M. Tratamento clínico. In: Dias JFP, Almeida HG. Glaucoma. Rio de Janeiro: Cultura médica;2000. p.319-33.

7. Pedroso L, Carvalho Jr ESC, Paranhos Júnior A, Prata Júnior JA, Mello PAA. Custo real do tratamento do glaucoma para o paciente. Arq Bras Oftalmol 1999;62:677-82.

8. Roizenblatt R, Freitas D, Belfort Júnior R, Höfling-Lima AL, Prata Júnior JA. Impacto econômico no tratamento do glaucoma: volume de gotas de colírios antiglaucomatosos brasileiros e norte-americanos. Arq Bras Oftalmol 2001;64:143-6.

9. Norvack GD, Evans R. Commercially available ocular hypotensive products: preservative concentration, stability, storage, and in-life utilization. J Glaucoma 2001;10:483-6.

10. Dicionário de Especialidades Farmacêuticas. $2^{\circ}$ edição. Rio de Janeiro: Publicações Científicas; 1972.

11. Dicionário de Especialidades Farmacêuticas. $11^{a}$ ed. Rio de Janeiro: Publicações Científicas; 1982.

12. Dicionário de Especialidades Farmacêuticas. $21^{a}$ ed. Rio de Janeiro: Publicações Científicas; 1992.

13. Dicionário de Especialidades Farmacêuticas 2002/03. 30ª ed. Rio de Janeiro: Publicações Científicas; 2002.

14. Guia Farmacêutico Brasíndice [online] [citado 2003 Mar 19]. São Paulo: Andrei Publicações Médicas. Disponível em: URL: http://www.brasindice. com.br/conheca.shtml

15. Silva LMS, Vasconcellos JPC, Temporini ER, Costa VP, Kara-José N. Tratamento clínico do glaucoma em um hospital universitário: custo mensal e impacto na renda familiar. Arq Bras Oftalmol 2002;65:299-303.

16. U.S. Department Of Labor, Bureau of Labor, Statistics. Consumer Price Index. [online] Washington, D.C.; 2002. [cited 2003 May 8]. Available from URL: ftp://ftp.bls.gov/pub/special.requests/cpi/cpiai.txt.

\section{Ao enviar un anrtigo panra publicação,

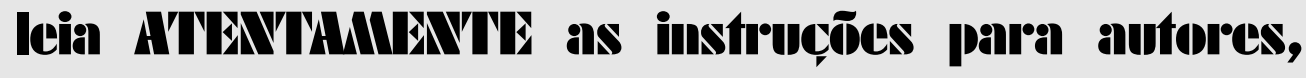 constante no final de cada fascículo.}

